\title{
E-learning for the new generations, a Web 2.0 approach
}

\author{
Hicham Hage and Esma Aimeur \\ Université de Montréal Canada
}

\section{Introduction}

E-learning has come a long way from being just a book-like content. Today, with the technological advances and the availability of higher bandwidth, E-learning became richer with multimedia content, more interactive and personalized. E-learning systems (also referred to as Learning Management Systems) and Intelligent Tutoring Systems (Brooks et al. 2006) are widely used to provide distant learning, and to complement the traditional classroom learning. Learning Management Systems have become very complex and include many utilities and tools, such as communication tools (chat, forum, email, etc.) to support electronic communication between tutors and learners, and among learners themselves, productivity tools (bookmarks, calendar, synchronization functionalities, etc.) to help learners be more productive, learner involvement tools (group work support, portfolio tools, etc.) to help the learners better interact with one another and course delivery tools (automated testing and scoring, course management, etc.) to help tutors with the delivery of course materials. Recently, E-learning has been going through new transformations into what is referred to as E-learning 2.0, with reference to Web 2.0 (Schauer 2005). The drive behind this change is the shift in education from being tutor-centered to becoming more learner-centered (Mccombs \& Vakili 2005) along with the support of Web 2.0 technologies. In tutor-centered education, the tutor is the active participant in the educational process and learners are considered as passive receptacles of knowledge, whereas in learner-centered education, the learners have access to various sources of knowledge and the tutor places more emphasis on what learners can contribute to the educational encounter. Similarly, Web 2.0 does not refer to an update or any technical specifications, but rather to changes in the ways software developers and end-users perceive and use the web. In the context of Web 2.0 web users are not just recipients of information, but actively participate in the creation of such information. The abundant Web 2.0 web pages and applications that can be used in the context of learning are starting to change the way tutors and learner perceive E-learning. Specifically, websites such as academia.edu, a social networking site for academics, promote networking between tutors and learners. Tutors have easy access to their peers, to collaborate and share knowledge, and learners can easily contact experts, and other learners for collaborative work and help. Additionally, many existing web pages and tools, such as Footnote.com, VoiceThread.com or Diigo.com, can help learners during the learning process. The combination of these tools, whether formal or informal, is referred to as a 
Personal Learning Environment (PLE) (van Harmelen 2006). The appeal of PLE for learners relies in the fact that they can choose the tools that best suit their preferences, whereas, the shortcoming of this is the lack of control of the tutor has on the educational validity and quality of the tools and information learners are accessing. On the other hand, Learning Management Systems provide a controlled learning environment, but lack the flexibility of PLEs. We argue that the best way is to combine both LMS and PLE into a single learning environment in order to take advantage of the control of LMS and flexibility of PLE, and we present SHAring REsources and Knowledge (SHAREK) (Hage \& Aimeur 2008), which provides a framework to combine a PLE within an LMS.

This chapter is organized as follows: Section 2 introduces Web 2.0, and details its three pillars: SOA (Service Oriented Architecture), RIA (Rich Internet Application) and Social Web. Section 3 illustrates E-learning 2.0 by defining learner centered education, and highlighting several Web 2.0 applications and technologies used for E-learning. Section 4 details the differences between Learning Management Systems (LMS) and Personal Learning Environment (PLE), and presents SHAREK. Section 5 concludes the chapter detailing the trends and challenges for combining LMS and PLE.

\section{Web 2.0}

Although the term Web 2.0 suggests a new version of the World Wide Web, it does not refer to an update or any technical specifications, but rather to changes in the ways software developers and end-users perceive and use the web. Indeed, the term Web 2.0 refers to a perceived second generation of web-based communities and hosted services (such as blogs, Wikis, etc.) which aim to facilitate creativity, and to promote collaboration and sharing between users. Table 1 formulates a sense of what is Web 2.0 by example:

\begin{tabular}{|l|l|}
\hline Web 1.0 & Web 2.0 \\
\hline Britannica Online & Wikipedia \\
Personal websites & Blogging \\
Publishing & Participation \\
Directories (taxonomy) & Tagging ("folksonomy") \\
Content Management Systems & Wikis \\
\hline
\end{tabular}

Table 1. Web 1.0 vs. Web 2.0 (O'Reilly 2005)

For instance, Britannica Online (http://www.britannica.com/) is the online version of the renowned Encyclopedia Britannica. The content of an encyclopedia is usually authored and validated by well established scholars, and the access to that content costs a certain amount of money, whether by buying the book collection, or an online membership. On the other hand, Wikipedia.com is a free multilingual encyclopedia, authored by the public at large. Wikipedia has 10 million articles written collaboratively by volunteers around the world (Wikipedia 2009). Almost all of its articles can be edited by anyone who can access the Wikipedia website.

In short, the following point summarizes the difference between Web 1.0 and Web 2.0: publishing vs. participation. Specifically, in Web 1.0 (publishing) the content is controlled by the publisher, and the users are just the recipient of the information. Whereas in Web 2.0 (participation) the users are no longer passive recipients of information, but are active 
participants in the creation of such information, participating in Wikis, tagging, rating, sharing, and/or referring websites. A recently published report (Lenhart et al. 2007) indicates that $64 \%$ of online teenagers in the US, ages 12 to 17, engage in at least one type of content creation. Moreover, both YouTube and Wikipedia are listed among the top 10 most visited sites by Alexa (Alexa 2008). Both sites rely heavily on user input.

There are three pillars to Web 2.0: the Social Web, Service Oriented Architecture (SOA) and Rich Internet Application (RIA).

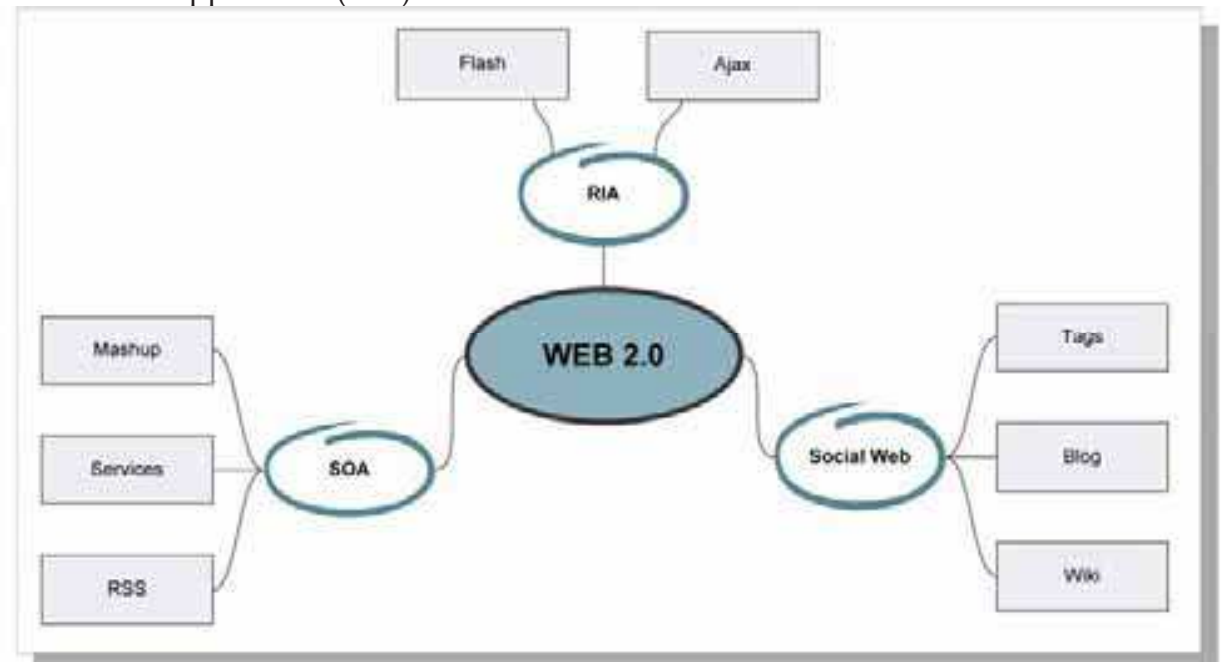

Fig. 1. The three pillars of Web 2.0 (Webilus 2008)

\subsection{Social Web}

The Social Web refers to the "social interactions" between the users of the web, and the resulting virtual "social groups". It allows users to share their writings, videos, photos, and more with their friends, family, colleagues, or the public at large. For instance, the Social Web includes simple publishing through a blog or a wiki. As such, in the case of the blog the owner of the blog and his faithful readers can become a social circle where the readers can comment on the blog posts, or each other's comments. Similarly, with the Wiki, the users who regularly visit, contribute to, or maintain the Wiki become a virtual social community centered on the Wiki.

Another aspect of the Social Web is the Social Networking Sites (SNS), where users can create a public or semi-private profile in order to stay in contact with other users of the site, referred to as Friends. Specifically, SNS such as MySpace.com or Facebook.com enable users to crate a profile page, add other users to their friends list, share photos, and other multimedia content. Other specialized SNS focus on certain aspects of social networking, such as LinkedIn.com which provides an environment for professional networking, and provides specific functionalities such as to recommend someone for a position or a job. Similarly, Academia.edu provides a social networking environment for academics, where researchers can connect and offers particular functionalities, such as to share research paper or to help locate other users with similar research interests.

The main drive behind the Social Web is collaboration and the harnessing of collective 
intelligence. Common features that exist in the Social Web, such as tagging, rating, comments and recommendation, exploit and share the knowledge and experiences of the users. As an example, we will consider social bookmarking sites, such as delicious.com or StumbleUpon.com. Such sites enable users to bookmark their favourite web sites. Moreover, users can share their favourite web sites with other users, or a community of friends. Additionally, users can add tags, comments, even rate these websites (such as thumbs up or thumbs down in StumbleUpon). Furthermore, users can search for web sites using the tags, or even receive recommendations based on their profile - StumbleUpon provides Collaborative Filtering (Burke 2002) recommendations based on the ratings of the users.

\subsection{Rich Internet Application}

Rich Internet applications (RIAs) are web applications that provide functionalities and interactions similar to desktop applications. Typically, RIAs are delivered through browser add-ons or directly through the webpage using for instance Ajax or Macromedia Flash. Consider the following application, Coolprieview (http://www.coolpreviews.com), to illustrate how RIA is delivered through a browser add-on. Coolpreview is designed to help users while browsing the web. Specifically, Coolpreview offers the users with the possibility to preview the page attached to with a link, without ever leaving the current page. For instance, consider the case of a user interested in information about the German Shepherd dog. After performing the search in Google, usually the user has one of three options: first, following each link returned by Google, then using the browser's back button to return to the search result page; second, opening each link in a new browser window, and third opening each link in a new tab. In contrast, Coolpreview opens for the user a virtual preview window, which displays the target website (Figure 2 in the middle). Additionally, Coolpreview offers several functionalities, including the possibility to display the next link on the page (or in this case the next web site), add the page to a temporary bookmark stack (Figure 2 on the right hand side), open the page in a new tab or even email the link. Hence, the user can visit several additional websites and perform various actions on them, without even leaving the initial page.

On the other hand, there is a multitude of web pages that illustrate the use of RIA, including web-based virtual computers, such as G.ho.st (http:// g.ho.st/). Such environments provide a virtual computer environment, accessible online using any browser, which provides the functionalities and tools or a regular computer, including disk space (5 Gbytes in the case of G.oh.st), a media player, and even an office suite to create, and store documents spreadsheets and presentations. 


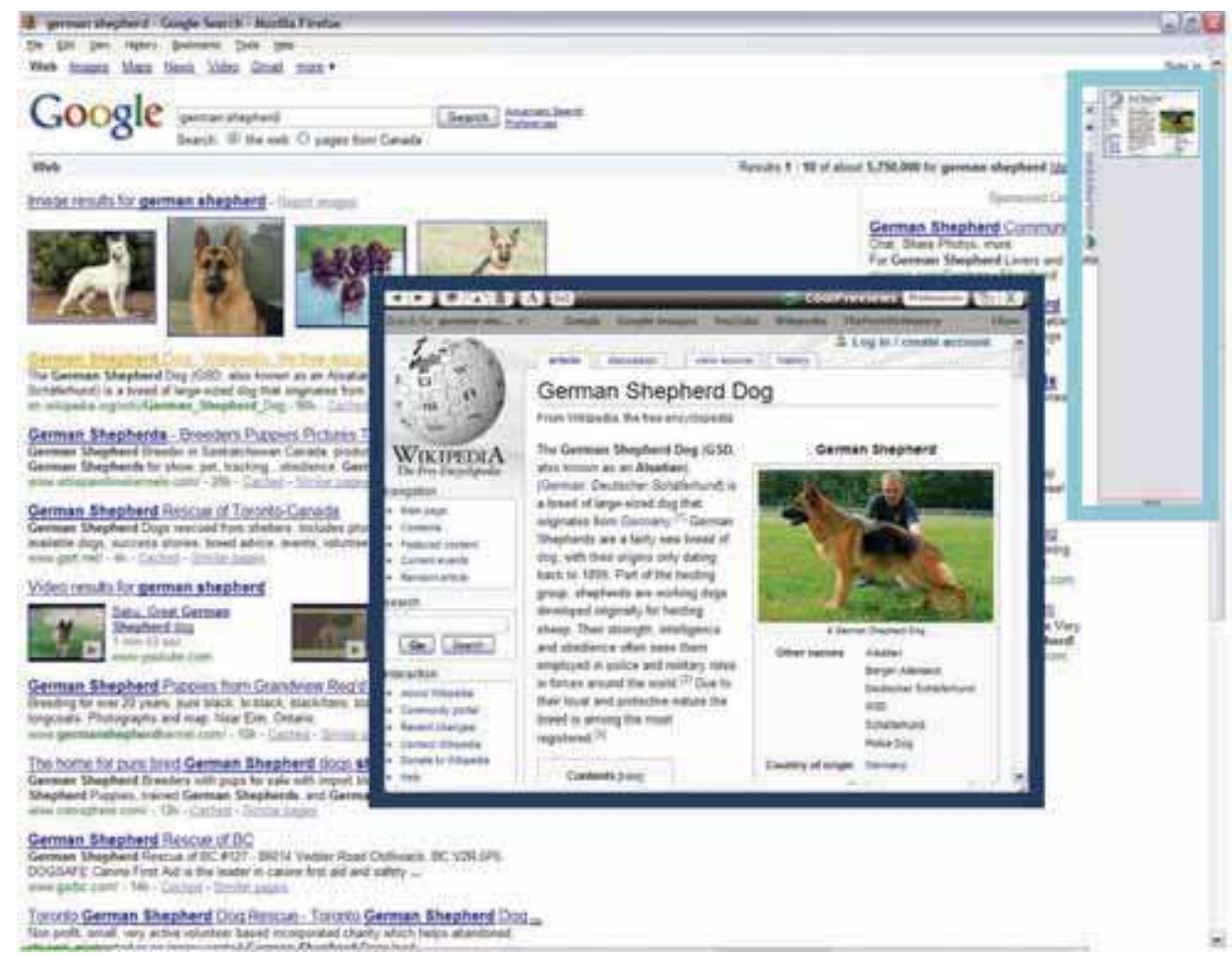

Fig. 2. Coolpreview

\subsection{Service Oriented Architecture}

Service Oriented Architecture (SOA) is an architectural style where the main goal is to relax the dependencies between various components and to achieve loose coupling. Specifically, a service is a task performed by the service provider to achieve a desired end result for a service consumer. Consequently, a service-oriented architecture is a collection of services (service providers and consumers), where these services communicate with each other. Such communication could be just simple data passing or it could involve two or more services coordinating to perform a certain activity. Note that the service provider can also be a service consumer. SOA usually employs a find-bind-execute paradigm as illustrated in Figure 3. The service provider registers in the directory, providing a detailed description of the service provided. The service consumer queries the directory to find a suitable service provider. When found, the service consumer sends a request to the service provider, who in turn sends the response to the service consumer. Some of the main advantages of using SOA are:

Reusability: In an SOA, a requesting application only needs to know the public interface of a desired service. Hence, the functions of an application are generally easier to access as a service in an SOA than in some other architecture. Consequently, integrating applications and systems as well as reusing their different components can be much simpler. 


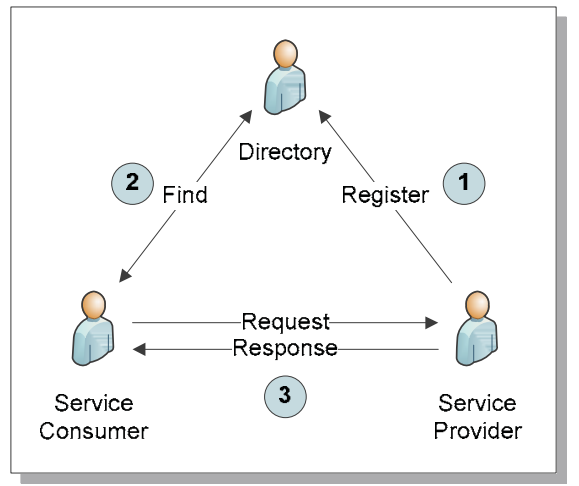

Fig. 3. Simple SOA - adapted from (Booth et al. 2004)

Interoperability: the interaction between loosely-coupled services implies widespread interoperability. In other words, within a SOA, the desired objective is for service consumers and service providers to communicate and understand each regardless of the platform they are on. This objective can be met by having a standard way of communication between services, a way that is consistent across various platforms, systems, and languages. Scalability: since services in a SOA are loosely coupled, the applications that exploit these services tend to scale easily, or at least easier than applications in a more tightly-coupled environment. This is due in large to the fact that there are few dependencies between the requesting application and the services it uses.

Flexibility: loosely-coupled services are typically more flexible than tightly-coupled applications. In a tightly-coupled architecture, the different components are usually strongly bound to each other, typically sharing semantics and libraries, and often sharing their state. This makes it difficult to evolve the application to keep up with changing requirements. The loosely-coupled and asynchronous nature of services in a SOA allows applications to be more flexible, and to easily evolve in order to adapt to changing requirements. The most common approach used to achieve the interactions between various services are the web services-based SOAs. A web service is a service that communicates with clients using a set of XML-based standard protocols and technologies, such as WSDL (Web Services Description Language), SOAP (Simple Object Access Protocol), and UDDI (Universal Description, Discovery, and Integration). The universality of the aforementioned protocols and technologies has made web services the most predominant approach to implementing a SOA. In short, WSDL (Web Services Description Language) an XML format used for describing a Web Services interface forms the basis of web services.

SOAP (Simple Object Access Protocol) is an XML-based protocol that enables applications to exchange messages and information over the internet. A SOAP message consist first of an Envelope that identifies the XML document as a SOAP message. The SOAP envelope contains an optional Header which provides information on authentication, encoding of data, or how the recipient should process the message. Moreover, the envelope includes the Body which contains the actual message or information.

UDDI (Universal Description, Discovery, and Integration) is a directory storing information about web services. In short, UDDI provides the definition of a set of services and supports the description and discovery of Web Services providers, the Web Services they make 
available, and the technical interfaces used to access those services. The idea is to locate organizations and the services they offer, much like using a phone book. Figure 4 highlights the SOA architecture presented in Figure 3 based on web services. Note that all the messages are exchanged using SOAP.

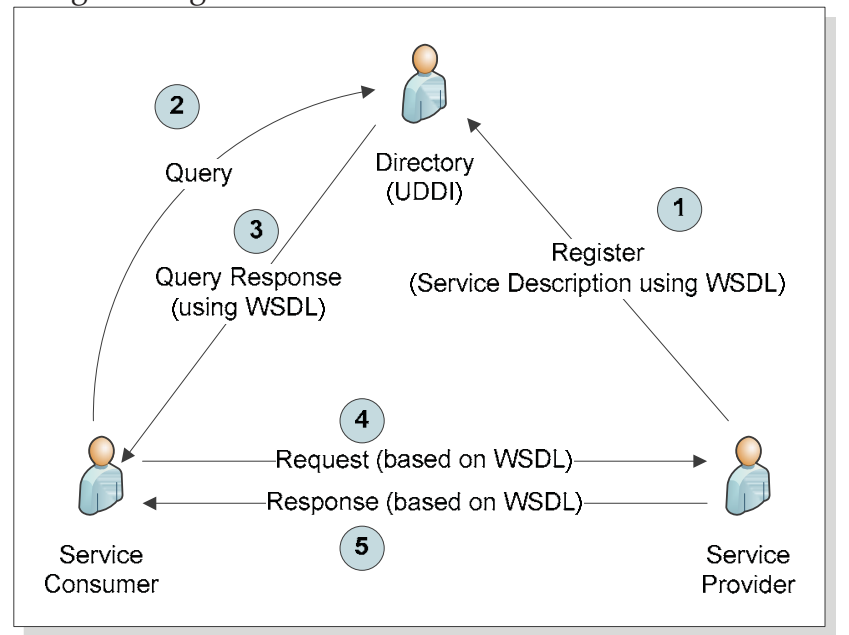

Fig. 4. Web service scenario

The flexibility and interoperability of SOA and web services has lead to a new type of web applications called Mashup. Specifically, a mashup describes a Web application that combines multiple services and/or data sources into one single application. Consider the following examples to illustrate a mashup. Woozor.com combines Google maps with information from weather.com in order to provide an interactive map wich displays weather forecast from around the world. Another example is Netzwelt.de, a German online magazine which combines free and legal promo MP3s along with Upcoming.org tour dates, Amazon CD reviews, YouTube videos and Akuma MP3 download store to provide exhaustive information for music fans.

\section{E-learning 2.0}

Now that Web 2.0 is clearly defined, let us define what E-learning 2.0 is all about. Similarly to Web 2.0, E-learning 2.0 does not refer to a new class of LMS (Learning Management Systems) or a new educational technology. Rather it is a natural consequence of changes in how tutors and learners perceive learning in general. Indeed, in recent years, education has been shifting from being tutor-centered, to being learner-centered. In tutor-centered education (Figure 5), the tutor is the active participant in the educational process and learners are 


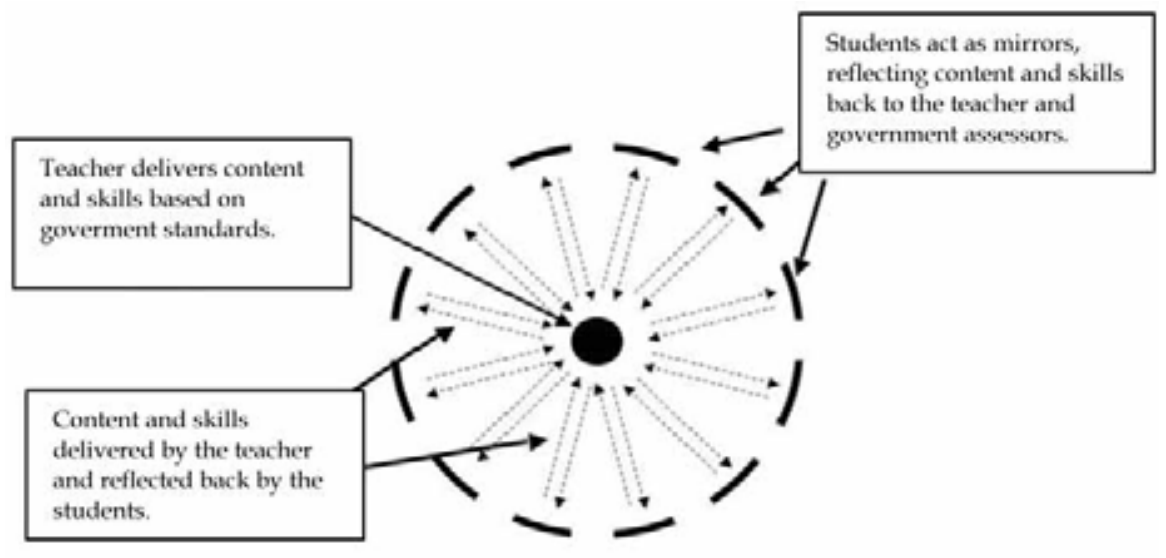

Fig. 5. Tutor-centered education (Webilus 2008)

On the other hand, in learner-centered education (Figure 6), the learners have access to a variety of knowledge sources and the tutor places more emphasis on what learners can contribute to the educational encounter. It is important to note that E-learning 2.0 is not a consequence of Web 2.0. Indeed, both share the same basic concept where the user/learner is not only a spectator and a simple consumer of information, but rather an active participant in the creation of such information. As such, one can view Web 2.0 tools and technologies as a natural recourse to achieve learner-centered education.

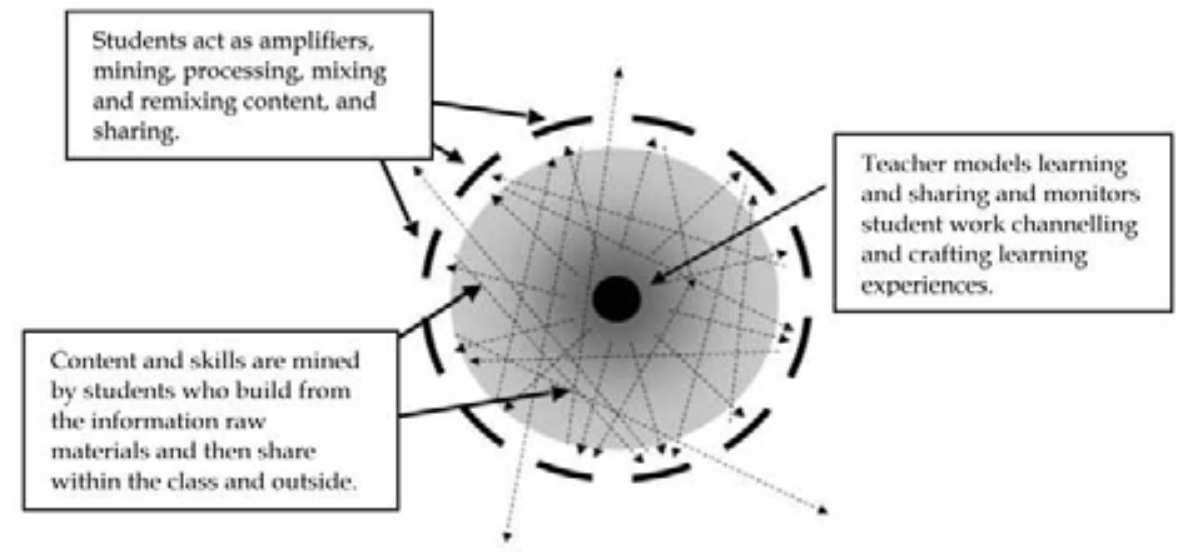

Fig. 6. Learner-centered education (Webilus 2008)

\subsection{Web 2.0 and E-learning}

This section provides some examples of "Web 2.0" tools and websites designed for and/or used in learning. For instance, a webcast consists of distributing media content over the using streaming media technology. A webcast may be distributed live or on demand. In essence, webcasting is "broadcasting" over the internet. A simple example of webcasting is a 
TV station that simultaneously streams over the internet the show being broadcasted on TV. On the other hand, a podcast is a series of media content made available via syndication, such as RSS. Dedicated software applications, known as podcatchers automatically identify and retrieve new available media files. In order to clearly understand the difference between a webcast and a podcast, consider the following example: you like to watch a TV show called "example show". If the show is aired once a week at a certain time, than you need to be home, in front of your TV at that specific day and time to watch the show. The only way to see an episode more than once is on reruns. This illustrates a live webcast. On the other hand, if that same show is available on demand TV, then you can watch the available episodes at your convenience. Nonetheless, you still need to be home in front of your TV. This is similar to an on demand webcast. Now, imagine the TV station has a delivery boy called "podcatcher" who will faithfully deliver to your house, every time a new episode of your show is ready, a DVD with that episode on it. In this case, you can watch any released episode at your convenience, whether on your home TV, or on a portable DVD player on the train on your way to work. This case illustrates the podcast.

The utility of webcasts and podcast in E-learning is very clear: tutors can either webcast their lectures live to students, or the lectures could be made available on demand or through a podcast. Note that a lecture can consist of various media, such as audio only, a slide presentation with audio, a recording of the tutor, etc.

Currently, webcasting and podcasting are being used in several universities worldwide (Shim et al. 2007). It is important to note that webcasting and podcasting are not just used by virtual universities, but also as a complement to lectures in traditional classrooms, for instance, Berkely makes publicly available webcasts of several courses (available at http:/ / webcast.berkeley.edu), consisting of either an audio recording of the tutor's lecture, a video recording of the tutor giving his lecture, or a slide presentation of the lecture with the explanations of the tutor.

Alternatively, wikis are websites that generally allow visitors at large to modify their content. Nonetheless, wikis generally can support authentication, such that certain members can modify only certain pages. This feature is important since it enable the use of wikis in group work assignments. Wikis offer the possibility of central access for all the users or limited user groups, which makes it an ideal choice for running projects, drafting documentations and other group work. As such, wikis are used to promote team work and collaboration between students (Raitman et al. 2005). Alternatively, wikis can also be employed by tutors to collaborate on creating learning content. For instance, wikiversity.org offers tutors the chance to collaborate and create freely available learning resources, where currently, on the English site of wikiversity, there are more than 10,000 pages available, covering various topics.

Similarly, SuTree.com and eduSLIDE.net offer both learners and tutors access to a variety of learning resources. Specifically, SuTree.com offers a variety of how-to videos, raging from learning how to whistle, to following a complete course watching MIT lectures. eduSLIDE allows tutors to create lessons (presentations) and group them into courses, making these courses available for learners.

Additionally, many existing "web 2.0" pages and tools can help learners during the learning process. For instance, Footnote.com allows students to access primary source documents and photos, and to easily create and post online history reports. Moreover, VoiceThread.com can be used by both tutors (to create lessons) and learners (for homework 
purposes) to upload pictures and create an audio narrative to go along with them. VisualThesaurus.com offers, as its name indicates, a visual thesaurus. Specifically the lookup word is presented in the center of the graph, and edges connect the lookup word with its synonyms. A color code is used on the edge connecting the word to its synonyms to indicate whether the synonym is a noun, verb, adjective or an adverb. Moreover, the edge connecting the lookup word with its antonym is presented differently. Wayfaring.com is a mashup that uses Google maps to list podcasts and webcasts from about 68 universities worldwide. wePapers.com allows users to share academic papers, raging from research papers, tutorials, lectures, to tests and exams. Moreover, users can comment, and even ask questions to the community about these papers. Another useful browser add-on is Diigo (http://www.diigo.com/). Diigo provides learners with the ability to highlight specific parts of webpages, add sticky notes and comments (private or public) to the highlighted sections or the whole page, and learners can share the highlights and notes with their Diigo social network.

\section{Combining LMSs and PLEs}

The proliferation of tools and websites such as listed earlier has led to the concept of Personal Learning Environment (PLE). PLE is a combination of tools and processes, whether formal or informal, which learners use to gather information, reflect on it and work with it. The appeal of PLE for learners relies in the fact that they can choose the tools that best suit their preferences. An interesting representation I came once across compares a Learning Management System (LMS) and a Personal Learning Environment (PLE) using the following analogy: an LMS is similar to a Swiss army knife containing a set of tools, some of which you might never used. On the other hand, a PLE is like having a box containing the tools you use, but most importantly tools that you chose and prefer. Indeed, although it might be more practical to fit a large set of tools into your pocket (Swiss army knife analogy), having only the specialized tools that you are comfortable with does have it advantages.

Many PLE advocates portray an LMS as being inflexible and used to control the learning and the learner, whereas a PLE is portrayed as easy to use, personalized, and liberated. In short, LMS is equivalent to controlling how you learn, whereas PLE corresponds to giving you control over how you learn. Although controlled and passive learning reduces self reliance and causes loss of curiosity and creativity, an uncontrolled education would create a shortage of certified labour and would introduce unqualified people into the labour pool. Ideally, a middle point between the flexibility of PLEs and the rigidity of LMSs would capitalize on the advantages of both worlds, while circumventing their weaknesses. Indeed, the driving concept behind learner centered education is to promote freedom and flexibility in learning, while maintaining some control (refer back to Figure 6). Specifically, this is where E-learning stands today (Figure 7). The Tutor delivers the learning content to the learner through the LMS. On the other hand, the learner has access to the controlled environment provided by the LMS as well as a PLE containing the set of his favourite tools and resources, which are external to the LMS. As such, the leaner can freely perform the learning activity, relying on the content and tools provided through the LMS, and on external uncontrolled resources through the PLE. In addition, the learner has access to both his personal social network (outside the LMS), and a peer network through the LMS. Note 
that some peers can also be part of the learner's external social network. In such a scenario, the tutor controls the curriculum (which courses and topics the learner must complete), and he can validate the learner's knowledge through assessments. On the other hand, the learner has the freedom to choose how to complete the learning activities: whether by solely using the content and tools provided through the LMS, by relying completely on his PLE, or a combination of both. In the last case, the LMS can be actually viewed simply as another component of the PLE.

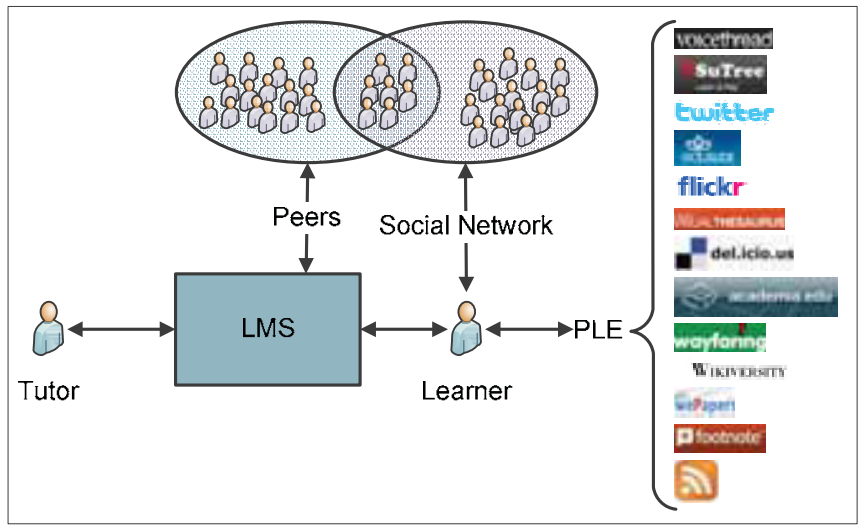

Fig. 7. Using LMS and PLE for education

\subsection{SHAREK: combining LMS and PLE}

Although this approach does bring together the advantages of LMS and PLE, it still presents some drawbacks, specifically to the learner. First, since there is no formal way to know what external resources the learner is accessing, the tutor cannot validate the content accessed and used by the learner. Hence, the learner may unwillingly access invalid content, which will induce him in error. Consequently the learner will be penalized during the assessment of his knowledge, which may cause conflicts with the tutor. Second, due to the large variety of resources and tools that could be part of a PLE, the discovery of new tools, as well as determining the most suitable tool (depending on the current needs) becomes a daunting task. In (Hage \& Aimeur 2008), we propose an approach that addresses both drawbacks mentioned earlier. The proposed approach is inspired by social bookmarking, and consists of adding support for a PLE within the LMS (Fig. 8). 


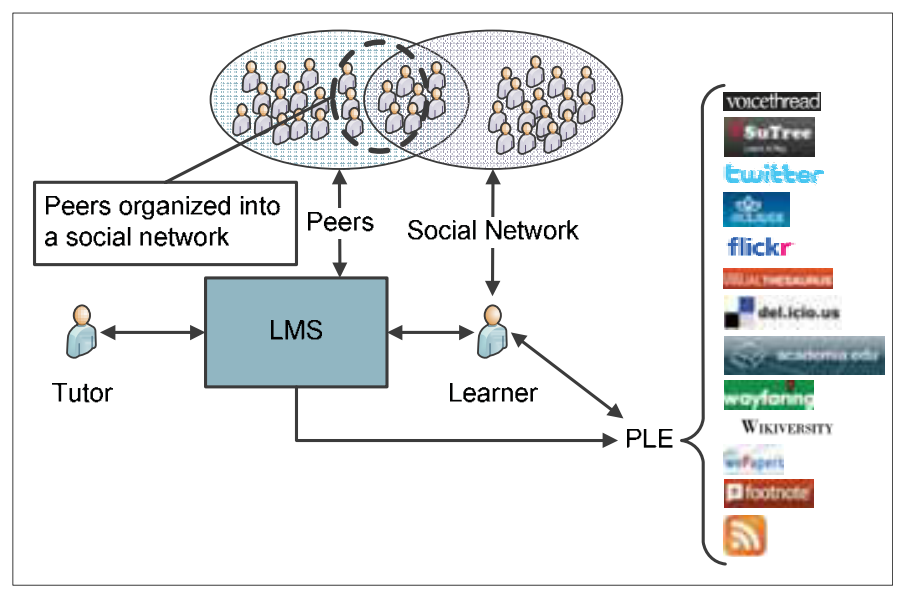

Fig. 8. Combining LMS and PLE

Specifically, learners can add learning resources into the LMS and could attach them either to a course, or a lecture within a course. As such, learners can access the PLE components by linking to them from within the course or lecture. For instance, a learner who relies on VisualThesaurus.com for an English course would add it as a resource to the course. Consequently, whenever the learner accesses this course, he can directly link to VisualThesaurus.com without having to search for it through a large set of bookmarks. Such an approach has many advantages. First, it enables learners to organize their PLE components with respect to the courses they are used in. Second, since these components are organized within the LMS, it enables the tutor to supervise, to some extent, the use of these components. Specifically, although the tutor cannot control or supervise the activities performed within the PLE, he can still know what components the learners are using in their PLEs. Hence, the tutor can take some actions accordingly: for instance, if he realizes that some learners are using Wikipedia for instance, he can make sure to advise them not to take that information for granted, since the content of wikis might be, in some cases, unreliable or biased. In short, the tutor can discourage and/or caution when questionable components are used in a PLE, and he can encourage and/or promote valid components. Third, this setup provides an ideal setting for the discovery of new components, whether for the learners or the tutor. Indeed, one can easily exploit this setup to allow learners to share their knowledge of learning resources. Specifically, in SHAREK (Hage \& Aimeur 2008) we propose the following process for sharing and discovery, highlighted in Figure 9. First, learners can add resources to SHAREK, where a resource could be an uploaded file (document, presentation, java applet, etc.) stored within the system for future access, or a link to an external webpage. Moreover, when a learner finds a useful resource, within SHAREK, which was contributed by another learner, he can add that resource to his favourites. Additionally, learners have the possibility to share a resource with their peers and/or friends. In this context, sharing a resource is similar to recommending the resource to peers and/or friends. Furthermore, RSS feeds are employed to facilitate the discovery of new resources. Specifically, RSS is a family of Web feed formats used to publish frequently 
updated content. Hence, each learner has his own feed, which is automatically updated to inform him of an add resource, favourite, or share events. In order not to be overwhelmed with updates, a learner can choose, for each of his friends and classmates, one or more event feeds to subscribe to.

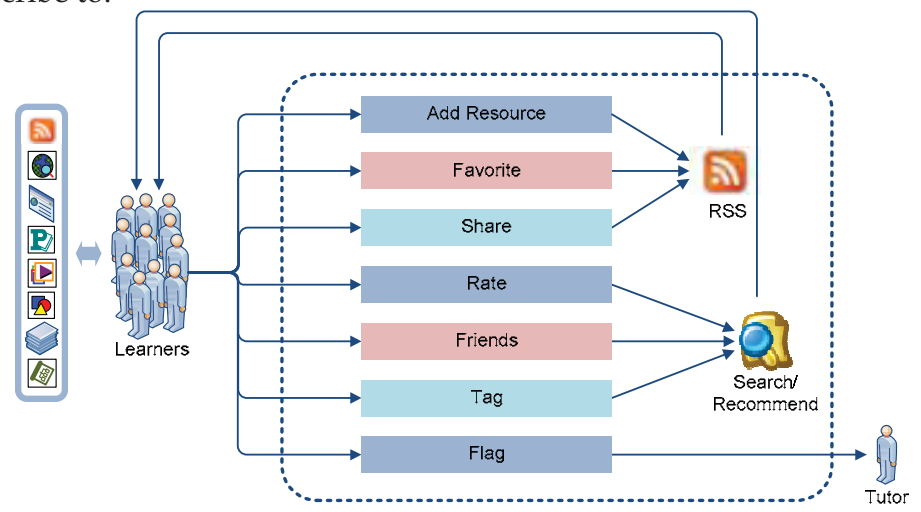

Fig. 9. SHAREK process

On the other hand, learners can rate the resources within SHAREK. A rating is a score (on a scale of 1 to 5) a learner gives to a resource. Within SHAREK, learners provide resources with an overall rating, as well as ratings with regards to its relevance to the course or lesson, its utility, and its clarity and ease of use. Such granularity (compared to using just an overall rating) is useful to evaluate the relevance, utility, and clarity of a resource. For instance, consider a resource that is very useful and easy to use, but was attached to an irrelevant course. When using only an overall rating, learners might give it a low rating because it is irrelevant to the course. Hence a learner who never used this resource would be inclined to consider it as a bad resource due to the low rating. With the granular ratings used within SHAREK, learners will have a better idea why the source was rated in such a manner. Additionally, this granularity in the ratings increases the accuracy of Collaborative Based recommendation techniques (Adomavicius \& Kwon 2007).

Moreover, learners can attach tags to the resource. Tags are keywords or terms associated with the resource to describe its content. Tags could be assigned by the contributor when adding the resource, or later on by other learners. Such a collaborative tagging approach, also known as folksonomy, has its strengths, and perhaps the most important is that it directly reflects the vocabulary of users. Indeed, a folksonomy, with its uncontrolled nature (in contrast to a Taxonomy), can adapt quickly to user vocabulary changes and needs. Learners can also flag a resource as being Inaccessible (resource cannot be accessed, broken link, unavailable file, etc.), Unrelated (Resource content is unrelated to lesson), Redundant (resource already exist) or Plagiarized (contributor asserts himself as the author of a resource created by someone else). When a resource is flagged, the flag will reflect within the system to advise learners accessing the resource, and a notification is automatically sent to the tutor to take the appropriate actions.

Additionally, learners can add selected peers and classmates to their list of friends. These friends are used in several contexts, including when specifying the RSS feeds the learner wants to subscribe to (as explained earlier), or when searching for new resources within 
SHAREK. Indeed, in addition to the RSS feed, SHAREK offers two other approaches to discover resources. The first approach is based on a CF (Collaborative Filtering) recommendation system. In short, a CF recommender system accumulates user ratings of items, identifies users with common ratings, and offers recommendations based on interuser comparison. Note that within SHAREK, we take advantage of the multiple criteria ratings of resources and use a multi-criteria collaborative filtering approach (Naak et al. 2009). Moreover, currently SHAREK performs the recommendation either at course level, or lecture level, where, in each case, only the resources attached at that level are considered. On the other hand, a content based search tool provides learner with another alternative to search for and find resources within SHAREK. Specifically, learners can search for resources by specifying one or more of the following criteria: language, rating, tags, date added, format, by the educational type, relation type, or even within their friends' favourite resources. These criteria are a part of the resources' information gathered and stored within SHAREK, and are inspired from, and based on the IEEE LOM (Learning Object Metadata) (IEEE Learning Technology Standards Committee 2002) standard. The data collected is divided into six categories.

The General category contains information such as the resource identifier, a unique id assigned by the system, the title, language and a short description of the resource, which are provided by the contributor at the time of adding the resource. Moreover, the general category contains the Tags associated to the resource, the learners' Ratings as well as the flags.

The Educational category contains information such as the type of the resource, whether the resource is a lecture, an exercise, a tool to help with the course, etc. Moreover, the educational category contains the Related To, and Relation Type information. The first describes which part of the course, or which lesson the resource is related to, and the second describes the relationship of the resource to the lesson or course: for instance, the resource could be a Java applet that illustrates the theory presented to the learner.

The Technical category contains information related as to what are the technical requirements to access the resource (such as plug-in or specific software), the format of the resource (a document, a java applet, a web site, etc.), the resource size (if applicable) and its location. It is important to note the content of location varies depending on the format of the resource. Indeed, if the resource is an uploaded file, then the location indicates where this file is stored. On the other hand, if the resource is located online (for example a website), the location, in this case, will contain the URL address. 


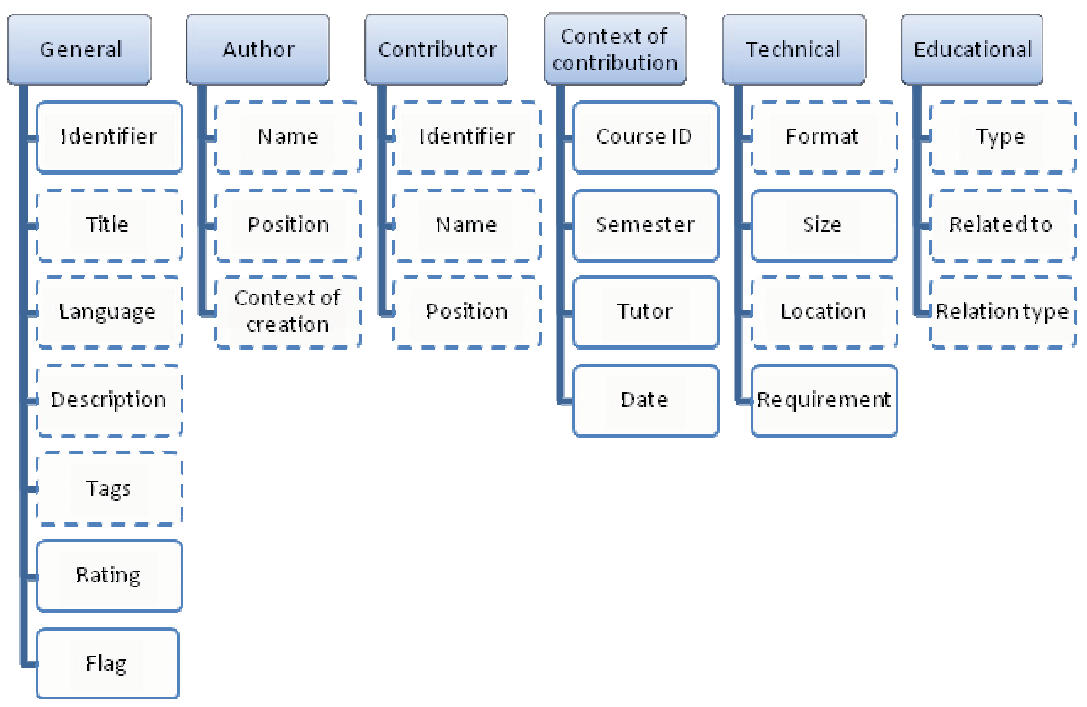

Fig. 10. Resource Metadata

The Contributor category contains the information to identify the learner who contributed, or added the resource. The contributor' unique identifier, as well as his full name and position are stored here. The position indicates the title of the contributor, whether he is a student, a teaching assistant or even a tutor.

The Context of Contribution category holds information about the context in which the resource was added, including the course id, the semester, the tutor and the date the resource was contributed. Such information is important and relates to context in which the resource was created.

Although the contributor could be the author of the resource, there are many situations when he is not. Thus, the Author category contains information about the actual author of the resource such as his name and position (student, tutor, etc.) and the context of creation of the resource (why the resource was created).

SHAREK was implemented and tested into a prototype platform that simulates an LMS, where learners follow a data structure course. 93 graduate and undergraduate students, from the computer science department at the University of Montreal, participated at testing the prototype. The participants highly rated the functionalities provided by SHAREK, and most reported that such an environment would encourage them to further share their knowledge.

\section{Challenges and future trends}

The change in education from tutor-centered to learner centered, along with the advent of Web 2.0 technologies is reshaping E-learning. Moreover, the proliferation of learning tools and the learners' appeal for PLE, created a competition between LMS and PLEs. Indeed, LMSs are constantly modified and upgraded to include such functionalities that are regularly used by learners and tutors as part of their PLE. Nonetheless, it is quite impossible 
for the LMS to conclusively win the race for many reasons. Indeed, if a LMS is to provide the large variety of utilities and tools used by learners, it will become very large and bulky, not to mention the high cost of development and maintenance. Moreover, the components of the PLE are usually specialized and dedicated for a certain task, thus outperforming the LMS at that specific task. Alternatively, a PLE cannot provide the stability and control required for proper learning. As such, the best and optimal approach is actually to combine the PLE within the LMS, thus successfully taking advantage of the stability and control of the LMS, while capitalizing on the flexibility of the PLE.

The approach proposed with SHAREK provides a framework to achieve this combination. The approach has many advantages, such allowing learners to share and discover new PLE components easily. Moreover, since these components are not physically part of the LMS, adding or removing them is a fairly simple task.

Nonetheless, this approach does have its limitations: since the PLE components are present outside the LMS, moving back and forth between the LMS and the PLE components might become, in many cases, tiresome. However, embedding the access to the PLE components from within the LMS (using for example approaches similar to CoolPreview) would resolve the issue. Other concerns include authentication and privacy. Indeed, many components and websites used within a PLE necessitate users to have an account and require authentication to give access to their services. It is clear that managing the multiple identities, which might grow quickly, is not a simple task. Additionally, existing research corroborates the need for learner privacy (Aimeur et al. 2007), yet having the learner's personal information duplicated within the PLE components presents some serious privacy concerns. For instance, having some of the learners information (demographic data, email, etc.) stored over the various PLE components increases the risk of unwanted access to such information. Nonetheless, mechanisms, such as OpenID (http:// openid.net) could be used to support the use of just one ID. Hence, including support within the LMS, and the PLE components for such mechanisms will limit the dissemination of learner's personal identification data over several platforms, while reducing and eliminating the need to manage several profiles. Nonetheless, other information could be still stored within the PLE components, which creates concerns with regards to the confidentiality, integrity and availability of such data. Indeed, many Web 2.0 applications, used as part of the PLE, are actually designed for sharing information purposes, consequently increasing the risk of unwarranted access to that information. Although we do advocate information sharing, there are cases where this access and sharing is not desirable and unwanted, for instance information about an ongoing homework or a project. Moreover, since the service might be temporarily unavailable or permanently shutdown, the learners' information might not be accessible or permanently lost.

Another approach to address these limitations would be to rely on the SOA (Service Oriented Architecture) paradigm, where the PLE components act as service providers and the LMS acts as the service consumer. In such a scenario, the learner does not need to leave the LMS in order to access the PLE components, instead, the LMS will request the service for the learner and provide the learner with the answer. Moreover, the learners' information could be stored within the LMS and only the necessary information is communicated to the service provider. For instance, consider Moodle (http: / / moodle.org), a popular open source LMS. The standard package deploys Moodle with the basic required components regularly used within an LMS. Additionally, for further enhancements and personalization, 
Moodle offers a vast collection of plugins and modules. Although most of these components are self contained, many rely on existing services and APIs: for example, one module exploits the Google API to offer text translations from within Moodle. Another module enables tutors to import learning objects from DOOR repositories into Moodle. Specifically, DOOR (Digital Open Object Repository) is an Open Source software used to create learning objects repositories. On the other hand, another module can be added to provide web services support in Moodle. In details, it enables Moodle applications to use web services in order to provide and/or consume services.

Yet, this approach has its limitations. For instance, the modules mentioned earlier are typically added by Moodle's system administrator, and not the learners. As such, whenever a learner needs access to a new module, a request must go through to the system administrator, which could become complicated for the learner as well as the system administrator, specifically within large educational institutions. Moreover, using web services to access external components might not suffice in many cases. Indeed, the LMS, or the service consumer, must have the proper support to properly consume the provided service, which might be complicated specifically in cases involving multimedia content. For instance, consider using web services to retrieve information from VisualThesaurus.com (introduced earlier in Section 3.1). In this case, the service provider, VisualThesaurus will reply sending the synonyms, antonyms and their respective types (whether it is a noun, verb, adjective or an adverb) in a textual XML format. As such, in order to properly consume the service, the LMS must be able to reconstitute the visual representation of the lookup word, and its relation to the synonyms and antonyms. Hence, in many cases the LMS will require implementing a new module, using an API (if it exists) in order to properly take advantage of the PLE components.

In summary, the first approach, proposed within SHAREK, provides more flexibility when it comes to adding or removing PLE components into the LMS, but is limited with regards to the confidentiality, integrity and availability of the learner's information stored within the PLE components. The second approach remedies this limitation, but is not flexible when it comes to adding or removing PLE components. In fact, in many cases it requires implementing new modules or plugins to be integrated within the LMS. Hence, we believe that the first approach, even though it represents some risks to the learners' information, remains more flexible, suitable and sustainable than the second approach.

\section{References}

Adomavicius, G. \& Kwon, Y. (2007). New Recommendation Techniques for MulticriteriaRating Systems. IEEE Intelligent Systems, 22, 3, (May/June 2007) page numbers (4855)

Aimeur, E.; Hage, H. \& ManiOnana, F. S. (2007). A Framework for Privacy-Preserving Elearning, Proceedings of Joint Itrust and PST conferences on Privacy, Trust Management and Security (IFIPTM 2007), pp. 223-238, July-August 2007, Moncton

Alexa. (2008). Retrieved January 2008, from http://www.alexa.com.

Booth, D.; Haas, H.; McCabe, F.; Newcomer, E.; Champion, M.; Ferris, C. \& Orchard, D.(2004). Web Services Architecture. Retrieved February 2009, fromhttp:/ /www.w3.org/TR/ws-arch/. 
Brooks, C. A.; Greer, J. E.; Melis, E. \& Ullrich:, C. (2006). Combining ITS and eLearning Technologies: Opportunities and Challenges, Proceedings of Intelligent TutoringSystems, 8th International Conference (ITS 06), pp. 278-287, June 26-30 2006, Jhongli,Taiwan

Burke, R. (2002). Hybrid Recommender Systems: Survey and Experiments. User Modeling and User-Adapted Interaction 12, 4, 2002) page numbers (331-370)

Hage, H. \& Aimeur, E. (2008). Harnessing learner's collective intelligence: a Web2.0approach to E-learning, Proceedings of 9th International Conference on Intelligent Tutoring Systems (ITS 2008), pp. 438-447, June 2008, MontrealIEEE Learning Technology Standards Committee (2002). Standard for Learning Object Metadata.

Lenhart, A.; Madden, M.; Macgill, A. R. \& Smith, A. (2007). Teens and Social Media. Retrieved January 2008, fromhttp://www.pewinternet.org/PPF/r/230/report display.asp.

Mccombs, B. L. \& Vakili, D. (2005). A Learner-Centered Framework for E-Learning Teachers College Record, 107, 8, 2005) page numbers (1582-1600)

Naak, A.; Hage, H. \& Aimeur, E. (2009). A Multi-criteria Collaborative Filtering Approachfor Research Paper Recommendation in Papyres, Proceedings of 4th InternationalMCETECH Conference on e-Technologies (MCETECH 2009), pp. 25-9, May 2009,Ottawa

O'Reilly, T. (2005). What Is Web 2.0. Retrieved January 2008, from http://www.oreillynet.com/.

Raitman, R.; Augar, N. \& Zhou, W. (2005). Employing Wikis for Online Collaboration in the E-Learning Environment: Case Study, Proceedings of 3rd International Conference on Information Technology and Applications (ICITA 2005), pp. 142-146, July 2005, Sydney

Schauer, B. (2005). Crucial DNA of Web 2.0. Retrieved January 2008, from http: / / adaptivepath.com/ideas/essays/archives/000547.php.

Shim, J. P.; Shropshire, J.; Park, S.; Harris, H. \& Campbell, N. (2007). Podcasting for elearning, communication, and delivery. Industrial Management \& Data Systems, 107,4, 2007) page numbers (587-600)

van Harmelen, M. (2006). Personal Learning Environments, Proceedings of 6th International Conference on Advanced Learning Technologies (ICALT 2006), pp. 815 - 816 July 2006, Kerkrade

Webilus. (2008). Retrieved January 2008, from http:/ /webilus.com/toutes-les-images. Wikipedia. (2009). Wikipedia, the free encyclopedia. Retrieved March 2009, from http: / / en.wikipedia.org/wiki/Wikipedia:About. 


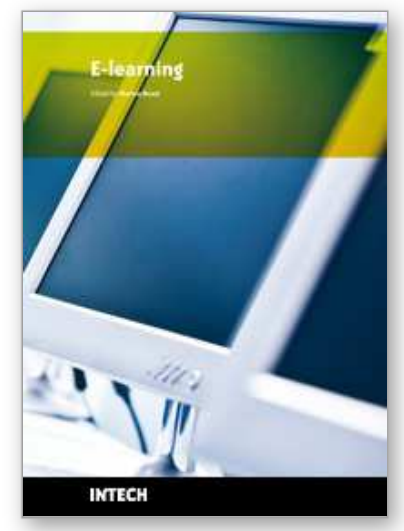

\author{
E-learning \\ Edited by Maja Jakobovic
}

ISBN 978-953-7619-95-4

Hard cover, 312 pages

Publisher InTech

Published online 01, February, 2010

Published in print edition February, 2010

E-Learning is a vast and complex research topic that poses many challenges in every aspect: educational and pedagogical strategies and techniques and the tools for achieving them; usability, accessibility and user interface design; knowledge sharing and collaborative environments; technologies, architectures, and protocols; user activity monitoring, assessment and evaluation; experiences, case studies and more. This book's authors come from all over the world; their ideas, studies, findings and experiences are a valuable contribution to enriching our knowledge in the field of eLearning. The book is divided into three sections. The first covers architectures and environments for eLearning, while the second part presents research on user interaction and technologies for building usable eLearning environments, which are the basis for realizing educational and pedagogical aims, and the final last part illustrates applications, laboratories, and experiences.

\title{
How to reference
}

In order to correctly reference this scholarly work, feel free to copy and paste the following:

Hicham Hage and Esma Aimeur (2010). E-learning for the New Generations, a Web 2.0 Approach, E-learning, Maja Jakobovic (Ed.), ISBN: 978-953-7619-95-4, InTech, Available from: http://www.intechopen.com/books/elearning/e-learning-for-the-new-generations-a-web-2-0-approach

\section{INTECH}

open science | open minds

\section{InTech Europe}

University Campus STeP Ri

Slavka Krautzeka 83/A

51000 Rijeka, Croatia

Phone: +385 (51) 770447

Fax: +385 (51) 686166

www.intechopen.com

\section{InTech China}

Unit 405, Office Block, Hotel Equatorial Shanghai

No.65, Yan An Road (West), Shanghai, 200040, China 中国上海市延安西路65号上海国际贵都大饭店办公楼 405 单元 Phone: +86-21-62489820

Fax: +86-21-62489821 
(C) 2010 The Author(s). Licensee IntechOpen. This chapter is distributed under the terms of the Creative Commons Attribution-NonCommercial-ShareAlike-3.0 License, which permits use, distribution and reproduction for non-commercial purposes, provided the original is properly cited and derivative works building on this content are distributed under the same license. 